\title{
IEEE-802.15.4-based low-power body sensor node with RF energy harvester
}

\author{
Thang Viet Tran and Wan-Young Chung* \\ Department of Electronic Engineering, Pukyong National University, Busan 608-737, Korea
}

\begin{abstract}
This paper proposes the design and implementation of a low-voltage and low-power body sensor node based on the IEEE 802.15.4 standard to collect electrocardiography (ECG) and photoplethysmography (PPG) signals. To achieve compact size, low supply voltage, and low power consumption, the proposed platform is integrated into a ZigBee mote, which contains a DC-DC booster, a PPG sensor interface module, and an ECG front-end circuit that has ultra-low current consumption. The input voltage of the proposed node is very low and has a wide range, from $0.65 \mathrm{~V}$ to $3.3 \mathrm{~V}$. An RF energy harvester is also designed to charge the battery during the working mode or standby mode of the node. The power consumption of the proposed node reaches $14 \mathrm{~mW}$ in working mode to prolong the battery lifetime. The software is supported by the nesC language under the TinyOS environment, which enables the proposed node to be easily configured to function as an individual health monitoring node or a node in a wireless body sensor network (BSN). The proposed node is used to set up a wireless BSN that can simultaneously collect ECG and PPG signals and monitor the results on the personal computer.
\end{abstract}

Keywords: Body sensor network, electrocardiography, photoplethysmography, low-power node, radio frequency energy harvester

\section{Introduction}

With the rapid developments of chip fabrication technology in the recent years, health monitoring nodes that are small, easy to wear, convenient to use and consume less energy have been developed widely [1]. An excellent study on a compact sensor node used an inertial sensor node based on the Sentilla Tmote Mini; its size was reduced tremendously, to only $4 \mathrm{~cm} \times 4 \mathrm{~cm}$ on four-layer PCB [2]. Another study was a wearable ECG acquisition system that was implemented with a planarfashionable circuit board and an ECG-monitoring system board $(5 \mathrm{~cm} \times 8 \mathrm{~cm})$ [3]. A highly integrated and miniaturized node with low power consumption for monitoring pulse and blood oxygen was comprised of three modules: a microprocessor control unit, wireless communication, and power system [4]. Another approach to ECG monitoring was an ultra-low power wireless sensor node, which had a maximum current consumption of only $27.04 \mathrm{~mA}$ [5]. The abovementioned research results are good choices for monitoring health signs. However, the power consumption or the size of those devices will be not sufficiently small to operate comfortably as a body sensor network (BSN) node. Alternatively, in this paper, an ECG front-end device with ultra-low power consumption and a PPG interface circuit are integrated with a microcontroller, a ZigBee wireless component, and a tiny antenna chip $(1.6 \mathrm{~mm}$

\footnotetext{
${ }^{*}$ Corresponding author: Wan-Young Chung, Department of Electronic Engineering, Pukyong National University, Busan 608-737, Korea. Tel.: +82-51-629-6223; Fax:+82-51-629-6210; E-mail: wychung@pknu.ac.kr.
}

0959-2989/14/\$27.50 @ 2014 - IOS Press and the authors. 
$\times 2.6 \mathrm{~mm}$ ). The proposed node has not only compact size and low power consumption property but also very low supply voltage. Therefore, the proposed design can be applied to the relatively new research field of energy harvesting, which has only existed for over ten years and has many applications in healthcare [6-9]. An enormous drawback of previous studies is limited operating duration [10,11]; these systems require batteries after working dozens of hours. This problem causes increased cost and inconvenience to users and pollutes the environment; therefore, some previous studies focused on the Peltier effect or the solar panel to prolong the lifetime, but the received powers were too low for healthcare application [7,9]. In this study, a 915-MHz RF energy harvester module is developed to charge the battery. When the proposed nodes work in the wireless BSN, all node batteries will be charged by the RF transmitter source (with a distance less than $1 \mathrm{~m}$ ) to prolong the lifetime. The wireless link uses the IEEE 802.15.4 standard, which is an attractive and convenient wireless standard for healthcare network systems [12-14].

The remainder of the paper is organized as follows. Section 2 is an explanation of the hardware design. Section 3 considers the software structure. Section 4 presents the results of monitoring the ECG and PPG signals using wireless sensor network and the current consumption results of the proposed design. Finally, section 5 is the conclusion.

\section{Hardware design and implementation}

Figure 1 shows the main components of the proposed node, including a microprocessor (MSP430), radio frequency component (CC2420 ZigBee), an ECG front-end circuit, a PPG sensor interface module, and a DC-DC booster circuit. The proposed node is powered by a DC supply with an input voltage supply ranging from $0.65 \mathrm{~V}$ to $3.3 \mathrm{~V}$ (using one or two AAA rechargeable batteries). An RF energy harvester module is used to receive RF energy within a distance of $1 \mathrm{~m}$ from the transmitter source to charge the battery.

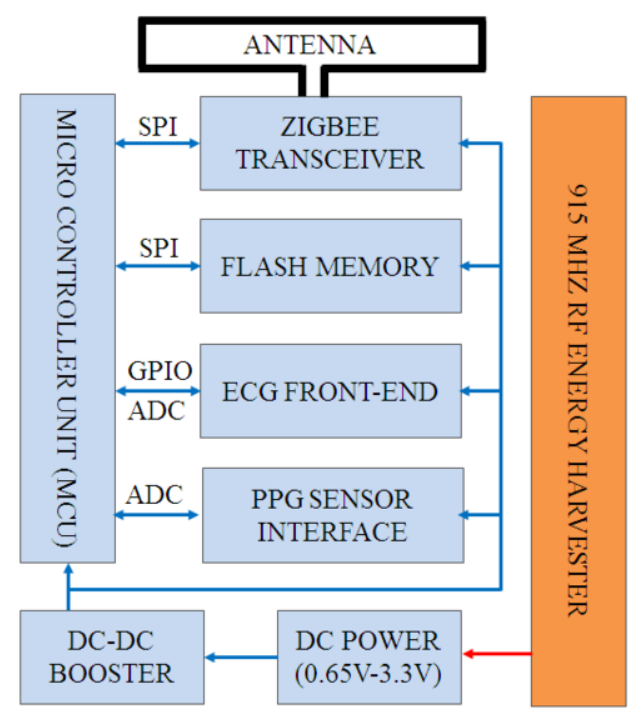

Fig. 1. Block diagram of the proposed node. 
Table 1

The current consumptions of the main components

\begin{tabular}{lll}
\hline Components & Power mode & Current consumption \\
\hline MCU-MSP430 & Active & $330 \mu \mathrm{A}$ \\
\hline \multirow{2}{*}{ RF-CC2420 } & Receive & $18.8 \mathrm{~mA}$ \\
\cline { 2 - 3 } & Transmit & $17.7 \mathrm{~mA}$ \\
\hline ECG-AD8232 & Typical & $170 \mu \mathrm{A}$ \\
\hline $\begin{array}{l}\text { DC-DC booster }- \\
\text { ISL9111 }\end{array}$ & Light load & $20 \mu \mathrm{A}$ \\
\hline
\end{tabular}
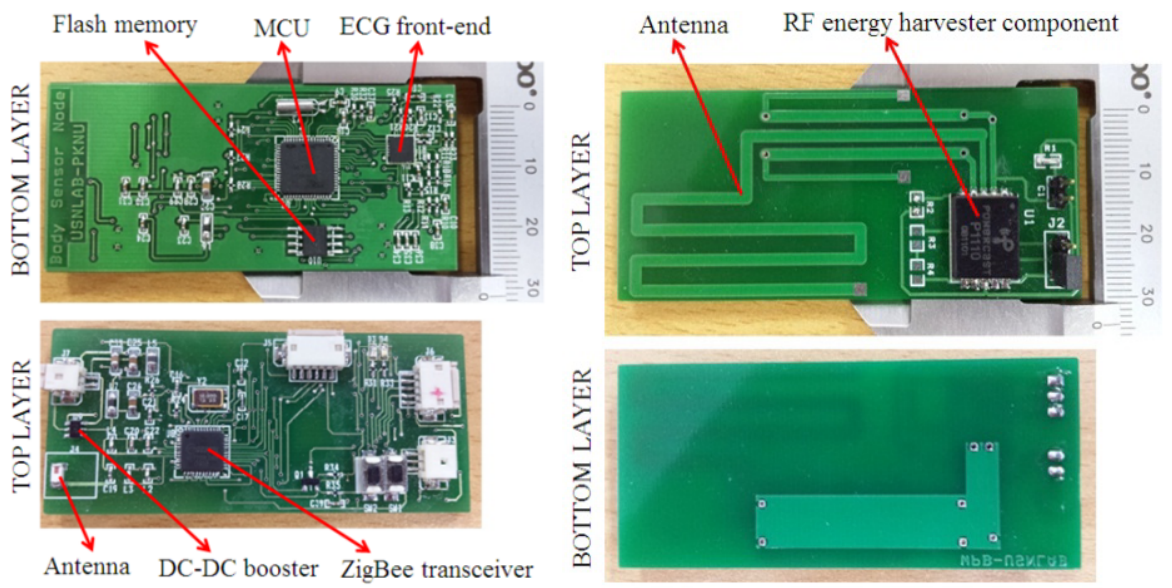

Fig. 2. Photograph of the proposed node. Fig. 3. Photograph of the RF energy harvester.

Table 1 shows the current consumption of the principal components of the node. The components have very low current consumption, and in active mode without a transceiver, the proposed node consumes very low power. Figure 2 shows two layers (top layer and bottom layer) of the proposed design; the PCB is $3 \mathrm{~cm}$ wide and $6 \mathrm{~cm}$ long. The RF energy harvester module is shown as Figure 3. This PCB measures only $3.3 \mathrm{~cm} \times 7.5 \mathrm{~cm}$ on two-layer PCB. It comprises a PCB antenna and RF energy harvester component.

The RF harvester part (P1110) is a product of the Power-Cast company in the USA, and it supports the health care monitoring systems. The P1110 has been optimized for operation in the $902-928 \mathrm{MHz}$ band and the maximum current power is $100 \mathrm{~mA}$ to charge the battery. The PCB antenna is modified from the original Power-Cast antenna $(1 \mathrm{~dB})$ to reduce the size, and is easily connected with the proposed platform.

The supply voltages of the studies on this technology are often around $3 \mathrm{~V}$, and these platforms have to be powered by two AAA batteries. Therefore, the power source occupies a large space, and when the power source approaches being empty, the system operation will become unstable. In this study, a DC-DC booster circuit, which is designed around ISL9111 chip, is integrated into the design to extend the supply voltage range and reduce the package size. The proposed course is a regulator with a very low input voltage ( $0.65 \mathrm{~V}$ to $3.3 \mathrm{~V})$ and a high-efficiency synchronous boost converter (up to $95 \%$ ). With this circuit, the proposed node can be powered by an AAA battery or energy harvesting. When 
the input voltage is lower than a threshold $(0.65 \mathrm{~V})$, the output voltage will assume the shutdown mode; therefore, the system will not fall into an unstable operational state.

The PPG sensor interface is designed for the RP520 device, which is a professional PPG sensor (Laxtha Co. Ltd., Korea). To collect an authentic PPG waveform, the selected cutoff frequency is approximately $8 \mathrm{~Hz}$ for a low-pass filter in this module. Whereas the ECG front-end circuit is built around the AD8232, which has ultra-low current consumption and small size $(4 \mathrm{~mm} \times 4 \mathrm{~mm})$. This module uses three electrodes: left hand (+), right hand (-), and right leg drive (GND) to gather the ECG signal. The standard ECG signal is by convention limited to $0.05-100 \mathrm{~Hz}$ [15]. However, this application presents a plan for monitoring the shape of the ECG waveform and eliminating the ECG baseline wander to ensure that a low-frequency band is chosen, in the range of $0.05-40 \mathrm{~Hz}$ [16]. In other words, there is a DC lead-off detection circuit inside the AD8232; this function indicates a disconnected electrode by setting the corresponding high signals $(\mathrm{LO}+$ for the left-hand electrode and LO- for the right-hand electrode).

\section{Software design}

The proposed node is supported by the nesC language under the TinyOS environment. TinyOS is an open-source operating system designed for low-power wireless sensor networks. Designers can easily develop wireless network applications to gather data without being concerned with unforeseen interactions [17-19]. NesC is a C-like language with features to reduce RAM and code size, enable significant optimizations, and help save time coding new software. With this programming language, programmers can easily write an application by defining new component modules and connecting existing component modules in a TinyOS library.

In this study, the ECG frequency band-width is $40 \mathrm{~Hz}$, and the PPG frequency band-width is $8 \mathrm{~Hz}$. According to the Nyquist sampling theorem, the sampling frequency is chosen as $100 \mathrm{~Hz}$ (two times greater than the signal frequency). To reduce transceiver time and prolong the battery lifetime, 10 data samples are collected and compressed into a data payload before transmission to the base station. The

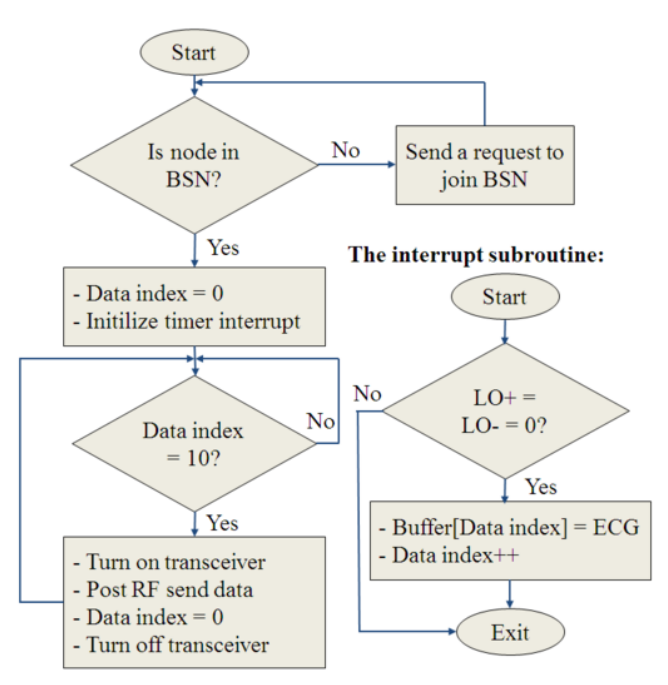

Fig. 4. Flow chart for collecting ECG signal.

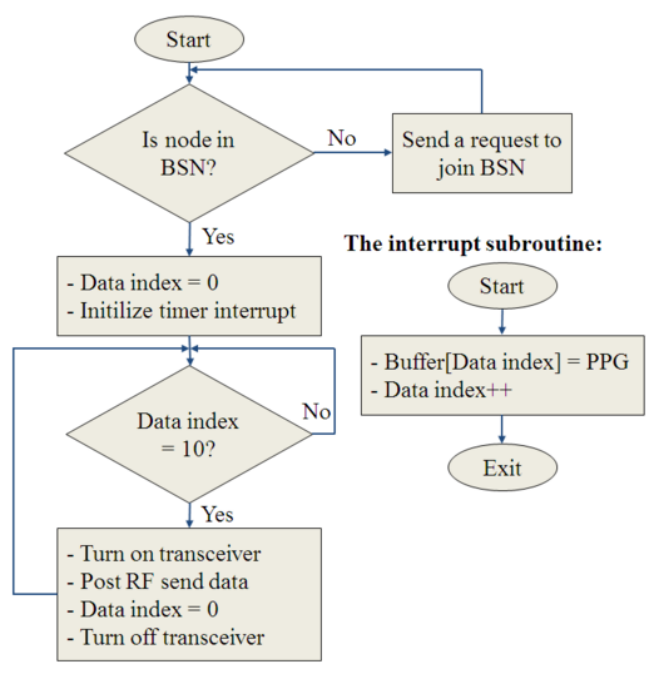

Fig. 5. Flow chart for collecting PPG signal. 
main program operates as shown in Figure 4 (for ECG signal) and Figure 5 (for PPG signal). After starting, each node checks itself to join the wireless BSN. This issue is not considered in detail in this research. When the proposed node has already joined the network, it activates the timer interruption task, which is $10 \mathrm{~ms}$ long (sampling frequency is $100 \mathrm{~Hz}$ ), and initializes a data pointer (Data index) as 0 to collect 10 data samples from the sensor. Before gathering the ECG data samples, the $\mathrm{LO}+$ and $\mathrm{LO}-$ signals have to be checked to ensure that these electrodes are connected to the subject. When 10 data samples are collected by the interrupt subroutine (Data index $=10$ ), a data frame is transmitted to the base station every $100 \mathrm{~ms}$ over the IEEE 802.15.4 standard.

\section{Results and discussion}

Figure 6 shows the power consumption of the proposed node while collecting an ECG signal in three cases (case1: one data sample in a frame, case 2: five data samples in a frame, and case 3:10 data samples in a frame).The results demonstrate that the proposed node can operate in the wide range of the input voltage, from $0.65 \mathrm{~V}$ to $3.3 \mathrm{~V}$. The power consumption is sharply reduced when $10 \mathrm{sam}$ ples are compressed into one data payload before transmission to the base station (using a flow chart in Figure 5). At approximately $1.5 \mathrm{~V}$ (equivalent to one AAA battery), the power consumptions of case 1 and case 2 were low, at approximately $14.5 \mathrm{~mW}$ and $17 \mathrm{~mW}$, respectively, whereas the power consumption was $27 \mathrm{~mW}$ in case 3 . At approximately $3 \mathrm{~V}$ (equivalent to two AAA batteries), the power consumptions of case 1 and case 2 were lower, at approximately $14 \mathrm{~mW}$ and $15.5 \mathrm{~mW}$, respectively, whereas the power consumption was $26.3 \mathrm{~mW}$ in case 3 . However, excessively increasing samples causes increased latency and requires significantly more channel time for communication.

An RF energy harvester is integrated into the proposed platform to charge the rechargeable battery $(1.5 \mathrm{~V} / 800 \mathrm{~mA})$. The battery lifetime without a charger is approximately 182 hours. Table 2 provides information on the received power of the RF energy harvester with a $1 \mathrm{~W}$ RF transmitter $(915 \mathrm{MHz})$. The system is able to harvest $1250 \mu \mathrm{W}$ at the distance of $1 \mathrm{~m}$, whereas the maximum harvested power

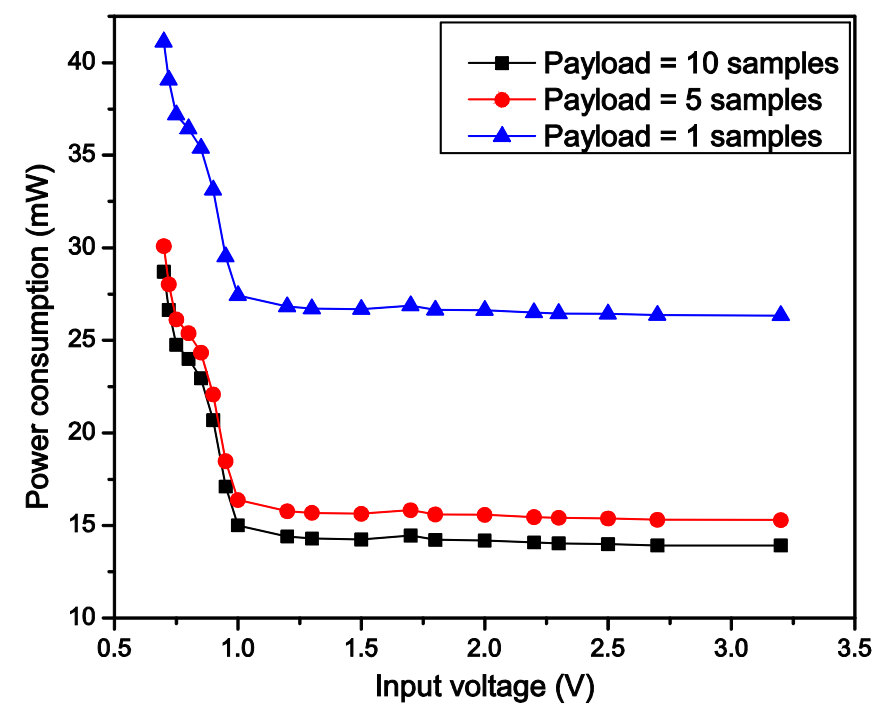

Fig. 6. Power consumption of the proposed node. 
Table 2

The received power of RF energy harvester

\begin{tabular}{lllll}
\hline No. & $\begin{array}{l}\text { Distance } \\
(\mathrm{cm})\end{array}$ & $\begin{array}{l}\text { Received pow- } \\
\text { er } \\
(\mathrm{mW})\end{array}$ & $\begin{array}{l}\text { Fully charging time } \\
\text { (hours) } \\
\text { (Battery: } \\
\text { mAh) }\end{array}$ & V/800 \\
\hline 1 & 5 & 33 & 36 & \\
\hline 2 & 10 & 30 & 40 & \\
\hline 3 & 25 & 17.5 & 69 & \\
\hline 4 & 50 & 2.5 & 480 \\
\hline 5 & 100 & 1.25 & 960 \\
\hline
\end{tabular}

from the flexible solar panel at 320 lux is only $76.65 \mu \mathrm{W}$ [9]. Referring to Table 2, if the distance from the proposed node to the RF transmitter is $50 \mathrm{~cm}$, the received power reaches $2.5 \mathrm{~mW}$; therefore, approximately 72 hours is added to the battery lifetime in working mode (equivalent to charging time is 182 hours). In standby mode, if the proposed node is placed nearly the RF transmitter (distance of 5 $\mathrm{cm}$ to $25 \mathrm{~cm}$ ), fully charging battery requires approximately 36 to 69 hours. The power consumption was also tested when the proposed node operated in different modes: active mode without RF, active mode while transmitting RF data, and active mode while receiving RF data (data rate was $115200 \mathrm{bps}$ ). The results are shown in Table 3. The main components have very low current consumption; therefore, in active mode without a transceiver, the current consumption was only $4.04 \mathrm{~mA}$, compared to 6.505 $\mathrm{mA}$ of [5] and $9.9 \mathrm{~mA}$ of [17]. In transceiver mode, the current consumption was slightly lower than the two others.

Table 3

Comparison of current consumptions from the different nodes

\begin{tabular}{llll}
\hline Operation modes & {$[17]$} & {$[5]$} & The proposed node \\
\hline $\begin{array}{l}\text { Active mode } \\
\text { without RF }\end{array}$ & $9.9 \mathrm{~mA}$ & $6.505 \mathrm{~mA}$ & $4.05 \mathrm{~mA}$ \\
\hline $\begin{array}{l}\text { Active mode with RF is sending } \\
\text { data }\end{array}$ & $29.6 \mathrm{~mA}$ & $27.4 \mathrm{~mA}$ & $23.5 \mathrm{~mA}$ \\
\hline $\begin{array}{l}\text { Active mode with RF is collecting } \\
\text { data }\end{array}$ & $27.3 \mathrm{~mA}$ & $20.31 \mathrm{~mA}$ & $19.5 \mathrm{~mA}$ \\
\hline
\end{tabular}
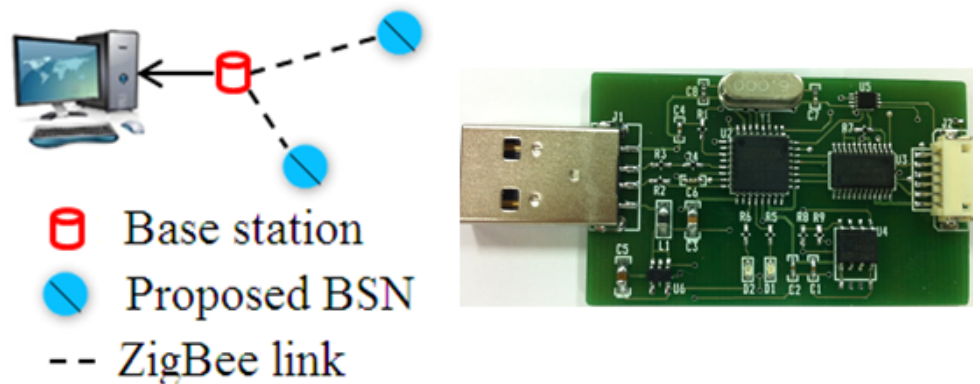

Fig. 7. Test environment of wireless BSN and a USB interface for the test. 


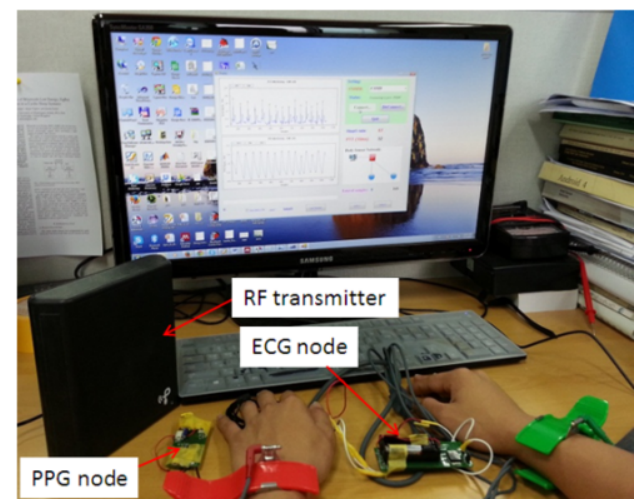

Fig. 8. Wireless BSN system for test.
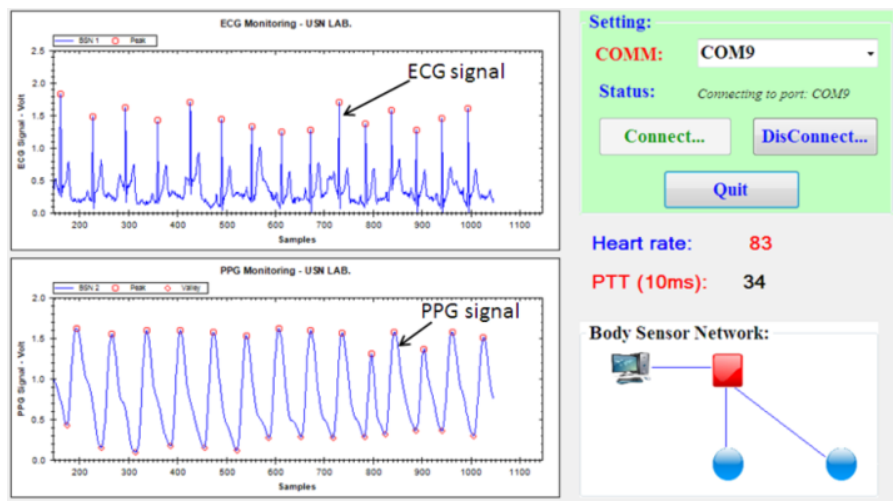

Fig. 9. A user interface for monitoring PPG and ECG signals.

During the operation, the proposed node operates in active mode without a transceiver; thus, that the average power consumption of the proposed node was significantly lower compared to that of the other nodes.

The proposed node also works as a wireless BSN node using the IEEE 802.15.4 standard. The system architecture is based on the star topology, as shown in Figure 7. It is comprised of a base station and two proposed BSN nodes. Each node can simultaneously sense two signals, but in this network application, there is one BSN node for gathering the ECG signal and another node for gathering the PPG signal. The base station includes a proposed node and a USB interface platform, which is shown on the right side of Figure 7. Figure 8 demonstrates the wireless BSN system based on the proposed node. The ECG node used three stainless steel electrodes to connect to the right hand, left hand, and left leg, whereas the PPG node used the RP520 PPG sensor. Two sensor nodes were placed near the RF transmitter to harvest the RF energy for charging the battery. Figure 9 shows the monitored results of PPG and ECG signals that are simultaneously measured from two different nodes in the wireless BSN.

\section{Conclusion}

The proposed node is convenient, has a low input voltage with a wide range, and features low power consumption. An RF energy harvester, which can harvest higher electrical power than solar panel or the Peltier effect, was also designed to prolong the lifetime of the node and charges the battery automatically in working or standby mode. In this study, the drawback of limited operating duration of BSN nodes has been solved. The proposed node is not only used as an individual node but also functions as a wireless BSN node. The DC-DC booster circuit was integrated into the proposed node; therefore, when the power source approaches being empty, the system will not fall into an unstable operational state.

\section{Acknowledgement}

This research was supported by Basic Science Research Program through the National Research Foundation of Korea (NRF) funded by the Ministry of Education (2012R1A1B3004140). 


\section{References}

[1] S.-J. Jung and W.-Y. Chung, A new wrist watch type healthcare monitoring system based on IP-USN, Sensor Letters 9 (2011), 393-398.

[2] Y.C. Kan and C.K. Chen, A wearable inertial sensor node for body motion analysis, Sensors Journal 12 (2012), 651657.

[3] J. Yoo, L. Yan, S. Lee, H. Kim and H.J. Yoo, A wearable ecg acquisition system with compact planar-fashionable circuit board-based shirt, IEEE Transactions on Information Technology in Biomedicine 13 (2009), 897-902.

[4] G.-L. Sun, J.-L. Yu, Y. Zhang and W.-X. Li, Design and implementation of sensor nodes for a wireless body area network, 4th International Conference on Biomedical Engineering and Informatics, 2011, 1403-1406.

[5] F. Sun, Z. Zhao, Z. Fang, D. Chen, X. Chen and Y. Xuan, Design and implementation of an ultra low power health monitoring node for wireless body sensor network, Fourth International Conference on Digital Manufacturing and Automation, 2013, 417-422.

[6] L. Huang, V. Pop, R. de Francisco, R. Vullers, G. Dolmans, H. de Groot et al., Ultra low power wireless and energy harvesting technologies-An ideal combination, IEEE International Conference on Communication Systems, 2010, 295300.

[7] M. Kawashima, T. Nakamura and K. Hata, Construction of healthcare network based on proposed ECG and physicalactivity sensor adopting energy-harvesting technologies, IEEE 15th International Conference on E-Health Networking, Applications and Services (Healthcom), 2013, 31-35.

[8] A. Nelson, J. Schmadt, W. Wilkins, J.P. Parkerson and N. Banerjee, System support for micro-harvester powered mobile sensing, IEEE 34th Real-Time Systems Symposium, 2013, 258-267.

[9] W.Y. Toh, Y.K. Tan, W.S. Koh and L. Siek, Autonomous wearable sensor nodes with flexible energy harvesting, IEEE Sensors Journal 14 (2014), 2299-2306.

[10] T. Martin, E. Jovanov and D. Raskovic, Issues in wearable computing for medical monitoring applications: A case study of a wearable ECG monitoring device, Fourth International Symposium on Wearable Computers, IEEE Comput. Soc., 2000, 43-49.

[11] C. Mo and J. Davidson, Energy harvesting technologies for structural health monitoring applications, 1st IEEE Conference on Technologies for Sustainability (SusTech), 2013, 192-198.

[12] J.S. Choi, Performance analysis of ZigBee-based body sensor networks, IEEE International Conference on Systems, Man and Cybernetics, 2010, 2427-2433.

[13] J.J. Garcia and T. Falck, Quality of service for IEEE 802.15.4-based wireless body sensor networks, 3rd International Conference on Pervasive Computing Technologies for Healthcare, 2009, 1-6.

[14] S.-J. Jung and W.-Y. Chung, Flexible and scalable patient's health monitoring system in 6LoWPAN, Sensor Letters 9 (2011), 778-785.

[15] C. Saritha, V. Sukanya and Y.N. Murthy, ECG signal analysis using wavelet transforms, Bulg. J. Phys. Journal 35 (2008), 68-77.

[16] Y. Xin, Y. Chen and W.T. Hao, ECG baseline wander correction based on mean-median filter and empirical mode decomposition, Bio-Medical Materials and Engineering 24 (2014), 365-371.

[17] X.J. Chen., M.Q.-H. Meng and H.L. Ren, Design of sensor node platform for wireless biomedical sensor networks, 27th Annual International Conference of the Engineering in Medicine and Biology Society, 2005, 4662-4665.

[18] Z. Sun, C.T. Yeh and W.-F. Wong, A UML 2-based hardware-software co-design framework for body sensor network applications, Design, Automation \& Test in Europe Conference \& Exhibition (DATE), 2011, 1-4.

[19] W. Kiing-Ing, A light-weighted, low-cost and wireless ECG monitor design based on TinyOS operating system, 6th International Special Topic Conference on Information Technology Applications in Biomedicine, 2007, 165-168. 